\title{
Computer Aided Detection for Low-Dose CT Colonography
}

\author{
Gabriel Kiss, Johan Van Cleynenbreugel, Stylianos Drisis, \\ Didier Bielen, Guy Marchal, and Paul Suetens \\ Faculties of Medicine \& Engineering, \\ Medical Image Computing (Radiology - ESAT/PSI), \\ University Hospital Gasthuisberg, \\ Herestraat 49, B3000 Leuven, Belgium
}

\begin{abstract}
The paper describes a method for automatic detection of colonic polyps, robust enough to be directly applied to low-dose CT colonographic datasets. Polyps are modeled using gray level intensity profiles and extended Gaussian images. Spherical harmonic decompositions ensure an easy comparison between a polyp candidate and a set of polypoid models, found in a previously built database. The detection sensitivity and specificity values are evaluated at different dose levels. Starting from the original raw-data (acquired at $55 \mathrm{mAs}$ ), 5 patient datasets (prone and supine scans) are reconstructed at different dose levels (down to $5 \mathrm{mAs}$ ), using different kernel filters and slice increments. Although the image quality decreases when lowering the acquisition mAs, all polyps above $6 \mathrm{~mm}$ are successfully detected even at $15 \mathrm{mAs}$. Accordingly the effective dose can be reduced from $4.93 \mathrm{mSv}$ to $1.61 \mathrm{mSv}$, without affecting detection capabilities, particularly important when thinking of population screening.
\end{abstract}

\section{Introduction}

Screening for colorectal polyps (precursors of colonic cancer in $90 \%$ of cases [1]) is currently advocated, based on the proven hypothesis that early detection and removal of colonic polyps results in a reduced mortality rate 2]. Amongst other methods Computed Tomographic Colonography (CTC) has been proposed as a screening candidate. It's main advantage compared to standard fiber optic colonoscopy is its minimal invasiveness and better patient tolerance [3]. Recent studies proved that CT colonography is an accurate method for detecting polyps above $5 \mathrm{~mm}$ in diameter.

The presence of ionizing radiation, inherent to CT acquisitions, hampers the usability of CT Colonography as a screening tool. The repeated patient scans (as part of the screening protocol) lead to dose accumulation, which can have harmful effects. Fortunately, the contrast between the colonic wall and the insufflated air or $\mathrm{CO}_{2}$ (Figure 1), employed to distend the colon is high enough and can be exploited to reduce the amount of dose per patient. Several studies [4] 5] have shown that despite significant image quality decreases, the 
polyp detection results remain unimpaired as compared to current protocols. One study [4] reported a dose reduction from $8.8 \mathrm{mSv}$ (median effective dose across several centers) to $3.6 \mathrm{mSv}$.

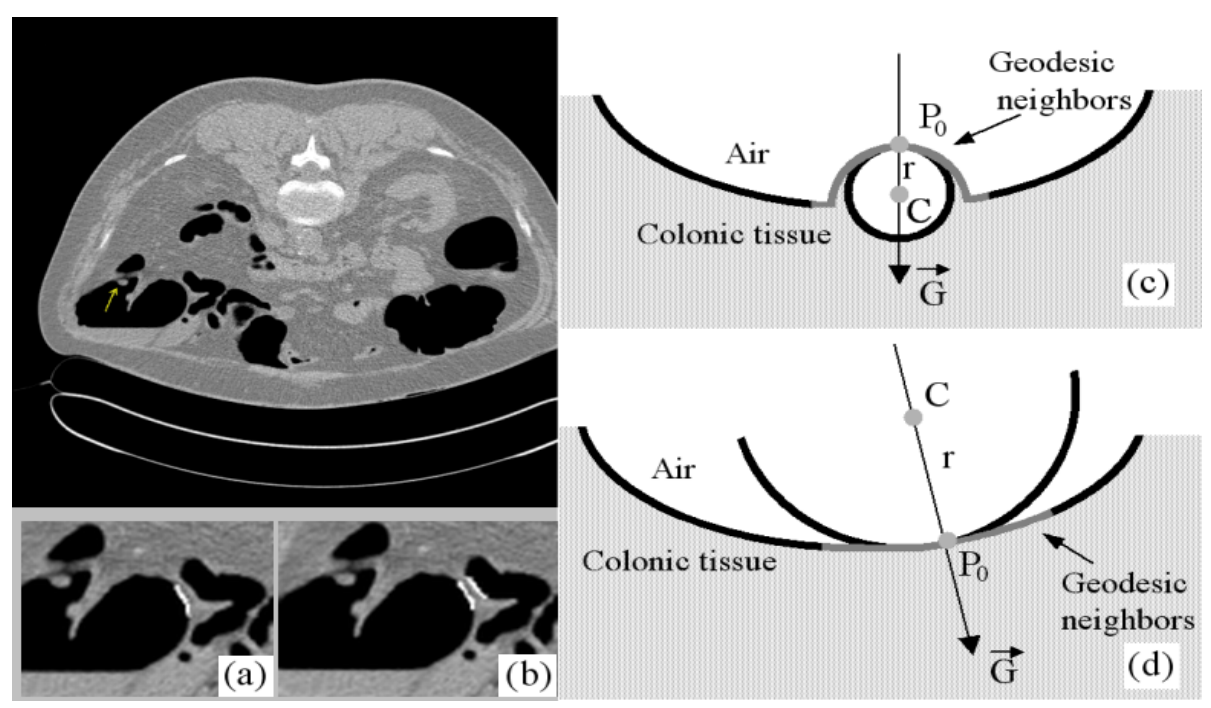

Fig. 1. CTC slice containing an $8 \mathrm{~mm}$ polyp. The difference between geodesic (a) and spatial neighbors (b) on a haustral fold is illustrated; the neighbors are depicted in white. The right column shows the fitting process and the resulting value of radius $r$ for a colonic polyp (c) and for normal wall (d).

During the last decade, Computer Aided Detection(CAD) methods have been proposed [6] [7] 8] 9] in order to increase reading sensitivity and to decrease interpretation errors and reading time associated with CTC. The input data for these algorithms is acquired using scan protocols predefined by the software of the scanner. To our knowledge, no study has established a relationship between the acquisition dose and the performance of the CAD technique. In this paper, the applicability of CAD to low-dose colonographic data is assessed. Additionally an alternative CAD method, directly applicable to low-dose datasets, is described.

Some of the previous methods look at the curvature along the colonic wall to detect initial polyp candidates [6] [7] 9], taking advantage of the ellipsoidal shape of polyps. Alternatively statistical pattern recognition and geometrical modeling [8] have been employed to detect polyps in CTC data. As described in 9] curvature based methods tend to be susceptible to noise, a balance between regularization (required to compute the curvature accurately) and the preservation of local image structures (improving overall sensitivity of the CAD method) has to be found. Finding an optimal setup is challenging, even for normal colonoscopic cases. The image noise also affects the computation process of features, thus negatively influencing statistically based approaches. 


\section{Methods}

The noise present in the low-dose data is handled by using robust fitting for generating initial polyp candidates and spherical harmonics for further analysis. Spherical harmonics [10] were chosen for detecting spatial trends and due to their ability to compensate for local perturbations given by noise.

The following subsections present the main steps of the proposed method; including a brief description of the segmentation step to obtain the colonic wall (section 2.1), the candidates generation (section 2.2), modeling of candidates using spherical harmonics and their classification into positive and negative cases (section 2.3).

\subsection{Segmentation}

The purpose of segmentation is to identify the colonic wall (found as the interface between colonic tissue and the air $/ \mathrm{CO}_{2}$ inside the colon). The method detailed in [8, consisting of classic region growing [11, with multiple seed points (to overcome collapsed regions), is used. The cumulative Laplacian histogram [12] has the ability to reveal optimal threshold values between air and colonic mucosa, especially important when using low-dose datasets (optimal threshold changes as the dose is lowered).

The neighboring voxels of a given structure have to be determined at each position along the colonic wall. A possible solution is given in 9 where a similarity weight between neighboring voxels is computed based on the vectorial inner product of normals. Our segmentation result is represented as an N-ary tree, based on neighboring relations between voxels. A local breadth-first transversal of the tree is sufficient to retrieve all neighbors with a geodesic distance (distance along the colonic wall) smaller than a threshold $T_{\text {dist }}$.

\subsection{Robust Sphere Fitting}

The first step of the classification algorithm is the identification of normal colonic wall, approximately $90 \%$ of the wall's surface. Assuming that colonic polyps have spherical shapes, we propose a sphere fitting method to discriminate normal colonic wall from colonic polyps.

The sphere fitting can be reduced to a linear process by introducing the following constraint: the center of the fitted sphere at any given location has to be along the gradient of the current point. Figure 1 presents the fitting process for a colonic polyp (c) and normal colonic wall (d). Analytical formulation yields:

$$
\begin{aligned}
& x_{c}=x_{0}-r * g_{x} \\
& y_{c}=y_{0}-r * g_{y} \\
& z_{c}=z_{0}-r * g_{z}
\end{aligned}
$$

considering $P_{0}\left(x_{0}, y_{0}, z_{0}\right)$ - the point on the colon wall (currently investigated), $C\left(x_{c}, y_{c}, z_{c}\right)$ - center point of the fitted sphere, $r$ - sphere radius (to be computed), $P(x, y, z)$ - neighboring voxels and $\boldsymbol{G}\left(g_{x}, g_{y}, g_{z}\right)$ normalized gradient in $P_{0}\left(x_{0}, y_{0}, z_{0}\right)$. 
The sphere equation and the normalization assumption are written as:

$$
\begin{aligned}
& \left(x-x_{c}\right)^{2}+\left(y-y_{c}\right)^{2}+\left(z-z_{c}\right)^{2}=r^{2} \\
& g_{x}^{2}+g_{y}^{2}+g_{z}^{2}=1
\end{aligned}
$$

From equations 1 and $2 r$ is computed as:

$$
r=\frac{\left(x-x_{0}\right)^{2}+\left(y-y_{0}\right)^{2}+\left(z-z_{0}\right)^{2}}{2 *\left(\left(x-x_{0}\right) * g_{x}+\left(y-y_{0}\right) * g_{y}+\left(z-z_{0}\right) * g_{z}\right)}
$$

Equation 3 can be seen as fitting a straight line through a set of data points, $N_{i}=r * D_{i}+e$, where $N_{i}$ and $D_{i}$ are the numerator and denominator of equation [3. $r$ - radius to be computed and $e$ - fitting error. Instead of solving equation 3 by a least square approach (which consistently overestimated the value of $r$ ), robust fitting was preferred. The following merit function is minimized iteratively, as described in [13:

$$
\Upsilon(e, r)=\sum_{i=1}^{n}\left|N_{i}-e-r D_{i}\right|
$$

Using robust estimation, outliers (generated by noise) are disregarded [13; additionally the size of the current structure is estimated implicitly. Geometric modeling will take the computed radius into account by adapting its parameters accordingly.

Each location on the colonic wall is labeled as normal if the value of the fitted radius is negative (concave wall patch) or greater than $T_{\text {radius }}$ (convex patch, however size is not interesting). The next step is to construct a Hough map, based on the remaining candidates, by back projecting with a radius given by $r$. The local maxima of the Hough map (identifying spherical shaped neighborhoods) are considered as input for the geometric modeling step.

\subsection{Geometric Modeling Using Spherical Harmonics}

To decide if a candidate location is a true polyp the most convenient approach is to match it to a set of known colonic polyps. However direct matching is not possible in practice, that is why polyps are modeled using shape descriptors and the corresponding descriptors are compared instead; the challenge becomes to find an accurate and fast measure of similarity with regards to all possible transformations. If translation and scaling can be easily addressed by normalization (to center of mass and to an average radius value for example), rotation is more time consuming. One possibility is to use PCA, however as mentioned in [10] PCA-alignment is negatively influenced by signal noise, since it mainly relies on the second order derivatives (the eigen values of the covariance matrix are computed using only second order information).

Due to their 'almost' spherical nature, polyps can be conveniently described by spherical functions. We have chosen a gray level appearance model (intensity profiles sampled along a sphere) and extended Gaussian images (mapping gradient information onto the unit sphere) to describe the shape of the polyp. 
Both descriptors are spherical functions defined on the $S^{2}$ sphere, unfortunately rotationally dependant ones. Using the framework proposed in [10, a spherical function $f(\theta, \phi)$, defined on the $S^{2}$ can always be decomposed into a sum of its harmonics:

$$
f(\theta, \phi)=\sum_{l=0}^{\infty} \sum_{m=-l}^{l} a_{l m} Y_{l}^{m}(\theta, \phi)
$$

The $L_{2}$ norm of the spherical harmonics is rotationally invariant, forming a complete orthonormal basis. The vector:

$$
\begin{aligned}
& S H(f)=\left\{\left\|f_{0}(\theta, \phi)\right\|,\left\|f_{1}(\theta, \phi)\right\|, \ldots,\left\|f_{l}(\theta, \phi)\right\|, \ldots\right\} \\
& \text { with } f_{l}(\theta, \phi)=\sum_{m=-l}^{l} a_{l m} Y_{l}^{m}(\theta, \phi)
\end{aligned}
$$

is invariant to all transformations, allowing to take the $L_{2}$ difference between two harmonic representations (up to a given bandwidth bw) of two different functions $f$ and $g$ as their similarity measure:

$$
S H(f)-S H(g)=\sum_{l=0}^{b w}\left(\left\|f_{l}(\theta, \phi)\right\|-\left\|g_{l}(\theta, \phi)\right\|\right)^{2}
$$

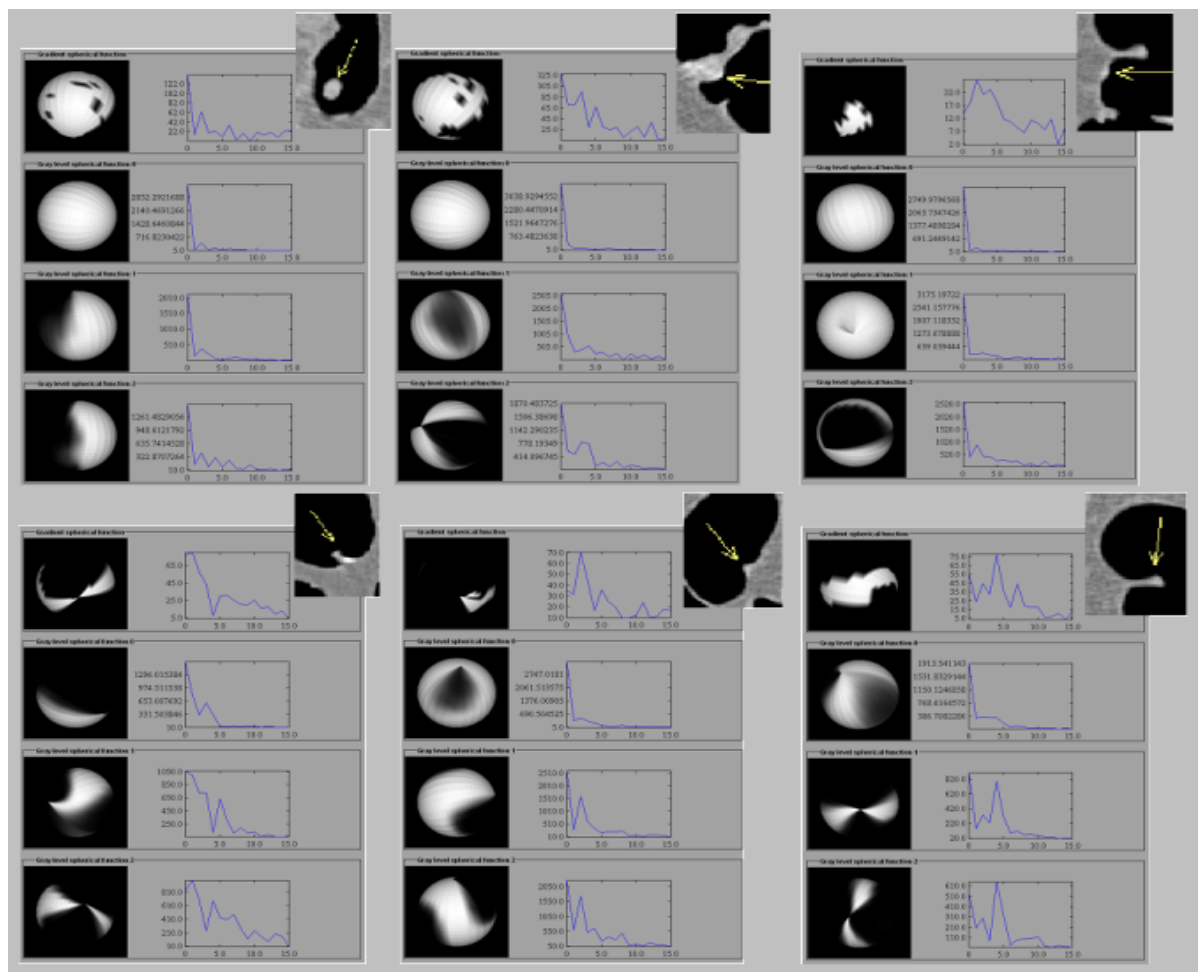

Fig. 2. Spherical harmonic decompositions for polyps (top row) and false positive cases (bottom row); for each case the spherical function and the harmonic decomposition are presented. The four spherical functions correspond, from top to bottom, to the extended Gaussian image and to the three intensity profiles at $r-2, r, r+2$. 
Based on our previously acquired cases (see Patient data) a database of model vectors as given by equation [6] was built. For each polyp three intensity profile decompositions were computed, one sampled on a sphere with radius $r$ (computed as described in section 2.2), and the other two at radii $r-2$ and $r+2$ respectively. The bandwidth $b w$ was taken as 16, the first 16 frequency components were included into our harmonic vector. This value was considered sufficient to capture the overall shape of colonic polyps. Additionally the extended Gaussian image around the polyp was computed and its spherical harmonic decomposition determined. Figure 2 presents typical feature vectors (gray level and extended Gaussian images) and their feature vectors given by equation 6 .

The database of models was clustered using hierarchical cluster trees (Matlab, Statistics toolbox). Clustering was required since polyps can have stalk, can be attached to the colonic wall or can lie on haustral folds, each having different signatures (as visible in figure 2).

\section{Patient Data and Results}

Fifty data-sets (cases D1) belonging to 26 patients, 25 normal and 25 with 28 polyps of various sizes ( 6 below $6 \mathrm{~mm}, 7$ between $6-9 \mathrm{~mm}, 11$ above $9 \mathrm{~mm}$ and 4 tumors) were considered as input for our CAD scheme. Additionally a set of 5 patients (cases D2) for which raw data was available and containing 12 polyps (4 between $6-9 \mathrm{~mm}$ and 8 above $9 \mathrm{~mm}$ ) formed the low-dose testing cases. All patients underwent CT colonography prior to conventional colonoscopy; standard colonoscopic preparation was given. Informed consent was obtained from all patients.

Cases D1 were acquired using a multi-detector CT (Multi Slice Helical CT; Volume Zoom, Siemens, Erlangen, Germany) using 4x1 mm detector configuration, $7 \mathrm{~mm}$ table feed per $0.5 \mathrm{~s}$ tube rotation, $0.8 \mathrm{~mm}$ reconstruction increment as well as 60 effective mAs and $120 \mathrm{kV}$. Somatom Sensation 16 (Siemens, Erlangen, Germany) scanned all the cases in D2, using a spiral mode with the

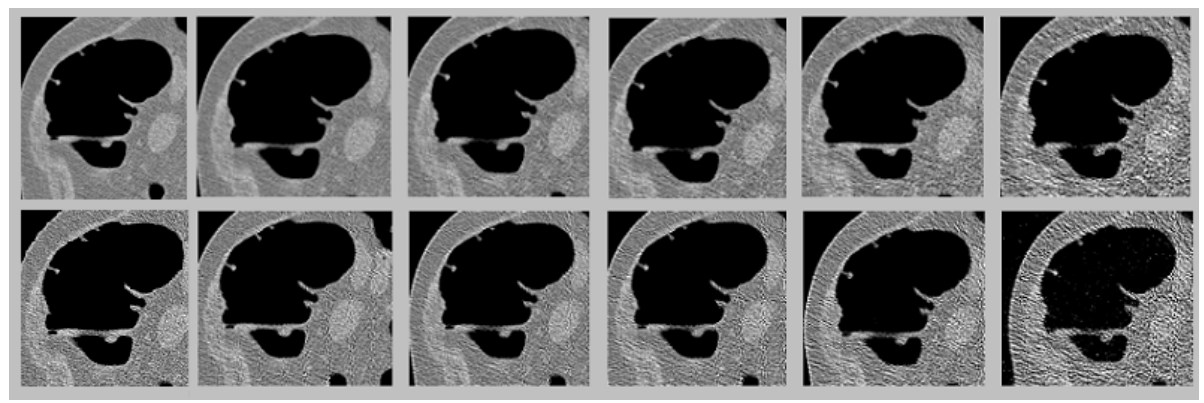

Fig. 3. Part of a CTC slice containing a $7 \mathrm{~mm}$ polyp, reconstructed at different $\mathrm{mAs}$ values: $55,45,35,25,15,5 \mathrm{mAs}$ (from left to right) and using two different kernels B40f-smoother (top row) and B30f-sharper (bottom row) 
Table 1. On the left simulated acquisition and reconstruction parameters. $C T D I_{w}$ (weighted CTDI value) and the effective dose are averaged over the 5 patients and taking into account both prone and supine acquisitions. On the right, results of the presented CAD algorithm on low-dose data, taking into account the best possible acquisition (0.8/1.0 Bf40) and all the reconstructed datasets (Overall).

\begin{tabular}{|c|c|c|c|c|c|c|c|c|}
\hline \begin{tabular}{|r}
$\begin{array}{r}\text { Voltage } \\
(\mathrm{kV})\end{array}$ \\
\end{tabular} & $\begin{array}{r}\text { Dose level } \\
(\mathrm{mAs})\end{array}$ & $\begin{array}{r}C T D I_{w} \\
(\mathrm{mGy}) \\
\end{array}$ & $\begin{array}{r}\text { Eff. dose } \\
(\mathrm{mSv}) \\
\end{array}$ & Dataset & \multicolumn{3}{|c|}{ Acquisition Sensitivity Specificity } & $\begin{array}{r}\text { False } \\
\text { positives }\end{array}$ \\
\hline 120 & 55 & 8.60 & 4.93 & $0.8 / 1.0 \mathrm{~B} 40 \mathrm{f}$ & $55 \mathrm{mAs}$ & $100 \%$ & $98.65 \%$ & 0.7 \\
\hline 120 & 45 & 7.06 & 4.05 & $0.8 / 1.0$ B $40 \mathrm{f}$ & $45 \mathrm{mAs}$ & $100 \%$ & $98.39 \%$ & 0.8 \\
\hline 120 & 35 & 5.54 & 3.18 & $0.8 / 1.0$ B $40 \mathrm{f}$ & $35 \mathrm{mAs}$ & $100 \%$ & $96.10 \%$ & 2.0 \\
\hline 120 & 25 & 3.92 & 2.25 & $0.8 / 1.0$ B40f & $25 \mathrm{mAs}$ & $100 \%$ & $94.67 \%$ & 2.8 \\
\hline 120 & 15 & 2.80 & 1.61 & $0.8 / 1.0 \mathrm{~B} 40 \mathrm{f}$ & $15 \mathrm{mAs}$ & $100 \%$ & $93.42 \%$ & 3.4 \\
\hline \multirow[t]{2}{*}{120} & 05 & 2.80 & 1.61 & $0.8 / 1.0 \mathrm{~B} 40 \mathrm{f}$ & $05 \mathrm{mAs}$ & $100 \%$ & $76.34 \%$ & 12.3 \\
\hline & & & & Overall & $55 \mathrm{mAs}$ & $100 \%$ & $95.94 \%$ & 2.7 \\
\hline Overlap & \multicolumn{2}{|c|}{ Slice increment } & Kernel & Overall & $45 \mathrm{mAs}$ & $100 \%$ & $94.88 \%$ & 2.8 \\
\hline $1.0 \mathrm{~mm}$ & \multicolumn{2}{|c|}{$0.8 \mathrm{~mm}$} & $\mathrm{~B} 40 \mathrm{f}$ & Overall & $35 \mathrm{mAs}$ & $100 \%$ & $94.84 \%$ & 2.8 \\
\hline $2.0 \mathrm{~mm}$ & \multicolumn{2}{|c|}{$1.5 \mathrm{~mm}$} & B40f & Overall & $25 \mathrm{mAs}$ & $100 \%$ & $85.80 \%$ & 7.6 \\
\hline \multirow[t]{2}{*}{$2.0 \mathrm{~mm}$} & \multirow{2}{*}{\multicolumn{2}{|c|}{$1.5 \mathrm{~mm}$}} & B50f & Overall & $15 \mathrm{mAs}$ & $100 \%$ & $80.07 \%$ & 10.2 \\
\hline & & & & Overall & $05 \mathrm{mAs}$ & $100 \%$ & $73.96 \%$ & 13.9 \\
\hline
\end{tabular}

following parameters: $16 \times 0.75 \mathrm{~mm}$ slice collimation, $11 \mathrm{~mm}$ table feed, 0.5 s rotation time, 55 effective mAs and $120 \mathrm{kV}$. All patients were scanned in both supine and prone positions, in average breath-holds of 19 seconds (16 slice) to 30 seconds (4 slice). The CAD process was carried out as follows: after the CT data was transferred to an offline workstation (Intel Pentium 2.4 GHz system), seed points were selected manually to ensure complete colonic segmentation. The segmentation threshold was determined automatically (section 2.1). The CAD pipeline presented in Section 2 was employed on the D1 cases to estimate optimal values for $T_{\text {dist }}, T_{\text {radius }}$ and also for computing a set of model spherical harmonics. The SpharmonicKit package (http://www.cs.dartmouth.edu/ geelong/sphere/) is used to compute the spherical harmonic decomposition. Taking conventional colonoscopy as standard of reference, ROC curves were computed. Parameter optimization consisted in maximizing the area under the ROC curve for values of $T_{\text {dist }}$ and $T_{\text {radius. }}$. A leave one patient out technique was preferred to compute the ROC curves for each possible parameter combination. The maximal value for the area under the ROC curve was: 0.995 , for $T_{\text {dist }}=3 \mathrm{~mm}$ and $T_{\text {radius }}=9 \mathrm{~mm}$. These optimal values of $T_{\text {dist }}$ and $T_{\text {radius }}$ were applied to the cases in $D 2$.

For the database D2 the influence of effective mAs, reconstruction kernel, slice overlap and increment, on the performance of CAD, was studied. Using the Somatom Noise+(V2.0) program (provided by Siemens, Forcheim, Germany), artificial noise (simulating a low-dose acquisition) was added to the original raw-data. The resulting raw datasets were imported into the scanner and 30 reconstructions per patient generated (Table 1). Figure 3 presents the same CT slice reconstructed at different dose levels. Due to limited space only results for the optimal setup (0.8 slice increment, 1.0 overlap, kernel B40f) and overall results (taking into account all 150 reconstructions) and are shown in table 1 .

The average computation time for the method is 78.18 seconds and can be further decomposed into: segmentation $7.97 \mathrm{~s}, \mathrm{~N}$-ary tree building $2.13 \mathrm{~s}$, candidates generation $27.38 \mathrm{~s}$, spherical harmonic computation and polyp extraction $40.70 \mathrm{~s}$ 


\section{Discussion and Conclusions}

The novelty of the paper is two-fold; first it proposes robust 3D models to describe colonic polyps, using the same framework, namely spherical harmonic decomposition, to capture both gray level and geometrical information. Second, the method was tested on low-dose data and proved the assumption that colonic polyps can be retrieved, without impaired sensitivity and specificity, even on data sets acquired at $15 \mathrm{mAs}$. Accordingly the dose reduction is significant, the effective dose can be reduced by a factor of 3 , very important when thinking of population screening and the associated risk of inducing cancer [4].

Several improvements to the proposed scheme can be introduced. First, our single scale statistical modeling (single bandwidth $b w=16$ ) can be transformed into multi-scale pyramidal modeling, to further reduce computation times. A modeling scheme based on spherical wavelets can be considered. Although better for describing smaller polyps, it is expected to be more sensitive to image noise [10].

Regarding low-dose data generation, the effects of reducing the $\mathrm{kV}$ of the original acquisition have to be tested. This will further reduce the effective dose. Phantom and real patient data can be used to evaluate this acquisition parameter. Finally, the use of denoising filters as proposed by Rust et al. 14] can be considered prior to CAD. While increasing the computation time, it could allow for even greater dose reductions.

To conclude, our results indicate that low-dose CTC CAD is viable. Sensitivity and specificity values remain high for the detection of clinically interesting cases (polyps larger than $5 \mathrm{~mm}$ ). Low-dose CTC CAD is a possible approach for colonic polyp screening, significantly reducing the chances of inducing tumors due to dose accumulation.

Acknowledgement. This work is part of the GOA/99/05: "Variability in Human Shape and Speech", financed by the Research Fund, K.U. Leuven, Belgium and supported by Siemens AG, Forcheim, Germany. The authors would like to thank Dr. Lutz Guendel for kindly providing the Somatom Noise+(V2.0) program.

\section{References}

1. Morson, B.C.: Factors Influencing the Prognosis of Early Cancer in the Rectum. Proc R Soc Med (1966) 59:607-8

2. Gazelle, G.S., et al.: Screening for colorectal cancer Radiology (2000) 215:327-335

3. Svensson, M.H., et al.: Patient acceptance of CT colonography and conventional colonoscopy: prospective comparative study in patients with or suspected of having colorectal disease. Radiology (2002) 222(2):337-345.

4. van Gelder, R.E., et al.: CT colonography at different radiation dose levels: feasibility of dose reduction. Radiology (2002) 224(1):25-33

5. Iannaccone R., et al.: Detection of colorectal lesions: lower-dose multi-detector row helical CT colonography compared with conventional colonoscopy. Radiology (2003) 229(3):775-781 
6. Summers, R.M., et al.: An Automated Polyp Detector for CT Colonography Feasibility Study. Radiology (2000) 284-290

7. Yoshida, H., Nappi, J.: 3-D Computer-Aided Diagnosis Scheme for Detection of Colonic Polyps. IEEE Transactions on Medical Imaging (2001) 1261-1274

8. Kiss, G., et al.: Computer Aided Detection in CT Colonography. LNCS 2878, MICCAI (2003) 746-753

9. van Wijk, C., et al.: On Normalized Convolution to Measure Curvature Features for Automatic Polyp Detection. LNCS 3216, MICCAI (2004), 200-208

10. D. Healy Jr., et al.: FFTs for the 2-Sphere - Improvements and Variations. Journal of Fourier Analysis and Applications 9:4 (2003), pp. 341 - 385.

11. Ballard, D.M., Brown, C.M.: Computer Vision. Prentice Hall (1982) 123-166

12. Wiemker R., Pekar, V.: Fast Computation of Isosurface Contour Spectra for Volume Visualization. Proceedings Computer Assisted Radiology and Surgery CARS (2001)

13. Press, W.H., et al.: Numerical Recipes in C - Chapter 15 Cambridge University Press (1992)

14. Rust, G.F., et al.: Noise/dose reduction and image improvements in screening virtual colonoscopy with tube currents of $20 \mathrm{mAs}$ with nonlinear Gaussian filter chains SPIE - Volume 4683 (2002) 186-197 